\title{
The quantitative neuropathology of schizophrenia
}

\author{
Patrick R. Hof · Christoph Schmitz
}

Received: 3 February 2009 / Accepted: 3 February 2009 / Published online: 27 February 2009

(C) Springer-Verlag 2009

Schizophrenia is a devastating neuropsychiatric disorder showing a variety of cognitive disturbances in attention, working memory, verbal production, response monitoring and inhibition, in short, almost all aspects of information processing and evaluation. Equally diverse are the putative mechanisms that translate such deficits into the anatomical substrates of schizophrenia pathology. Alterations in brain wiring and connectivity have long been suspected to play a key role in the development of schizophrenia. The resulting disorganization of neuronal systems in specific regions of the cerebral cortex and in certain subcortical structures may represent an anatomic and functional substrate for the constellation of symptoms that are typically observed in schizophrenic patients. To date, many studies have reported consistent and at times severe changes in the gray matter in schizophrenia and have paid attention to the connectivity of specific subsystems and particular populations of neurons. Reported abnormalities concern not only the numbers or densities of neurons but also their local microcircuitry and their distribution. Thus, altered densities and distribution of various populations of neurons have been reported in the prefrontal cortex in schizophrenia. Subclasses of interneurons are known to display abnormal localization in the subcortical white matter of the prefrontal cortex suggestive of cortical developmental pathology. Other data point to alter-

P. R. Hof $(\bowtie) \cdot$ C. Schmitz

Department of Neuroscience, Mount Sinai School of Medicine, Box 1065, One Gustave L. Levy Place,

New York, NY 10029, USA

e-mail: patrick.hof@mssm.edu

C. Schmitz

Division of Cellular Neuroscience,

School for Mental Health and Neurosciences,

Maastricht University, Maastricht, The Netherlands ations of calretinin-containing interneurons, abnormal axonal terminals from inhibitory neurons, reduced gene expression of markers of inhibitory neurons, and to pathology of chandelier neuron axons endings on pyramidal cells in the prefrontal cortex of schizophrenic patients. Neuronal packing densities have been shown to be elevated in the prefrontal cortex of schizophrenic cases with a severe reduction in size of large layer III pyramidal cells, indicating that profound cellular attrition occurs during the progression of the disease. It has been proposed that such pathology of large projection neurons could be the result of altered inhibitory activity and an expression of excitotoxic damage. Comparable changes have been reported in the entorhinal cortex with abnormalities in neuronal distribution within the layer II clusters, as well as dendritic alterations, cell size reduction, and in certain subdomains of the entorhinal cortex, lower neuronal densities. A deficit in synaptic transmission has also been suggested in schizophrenia based on reduced synaptophysin immunoreactivity in the prefrontal cortex as well as a severe impoverishment of the dendritic structure of pyramidal neurons and loss of dendritic spines. Finally, the thalamus has been the object of many studies of neuronal numbers showing that schizophrenia is accompanied by a variable loss of neurons at the nucleus or subnucleus level, suggesting a certain degree of regional specificity in the pathology that may differentially affect specific sets of connections to the prefrontal cortex. It is worth noting that these alterations are specific to certain neural systems, as many regions of the brain do not show such changes.

The concept of disrupted connectivity and abnormal synaptic transmission is key to our understanding of the cognitive and psychotic symptoms in schizophrenia. The involvement of the thalamoprefrontal pathway, together with the morphological alterations of prefrontal pyramidal 
neurons that are known to be the target of these thalamic projections, is interesting in the context of the cognitive changes and particularly the impairment in working memory known to occur in schizophrenia, as these functions depend, in part, on the integrity of the projections from the mediodorsal nucleus of the thalamus to the prefrontal cortex. It is worth noting that cognitive deficits can be severe in schizophrenic patients, but are unlikely to be directly the consequence of Alzheimer-like pathologic changes, as usually few neurofibrillary tangles and senile plaques (and, as recently reported, Lewy bodies) are found in the brains of such cases, indicating that the cognitive changes are probably the result of alterations that are specific to schizophrenia. The relationship between the involvement of thalamocortical systems and the positive and negative symptoms in schizophrenia is less clear. However, misrouting of corticocortical systems such as those linking the temporal cortex to the prefrontal cortex, as well as the intrinsic organization of the cerebral cortex may be crucial events in the establishment and severity of the symptomatology. Much evidence from analysis of schizophrenic brains using genomics and microarrays further support the notion that dramatic changes in cortical connectivity and synaptic transmission take place in schizophrenia. In particular, studies reported a decreased expression of a group of genes, encoding proteins, involved in the regulation of presynaptic function. These results suggest that a rather subtle molecular pathology takes place in the development of schizophrenia, as it is unlikely that the local changes in neuronal densities (but not in the actual total number of neurons) account for these findings. Other independent data sets found that the expression of several myelin-related genesis significantly reduced in the postmortem brain tissues of schizophrenic patients compared to controls. This is particularly important considering that genetic studies of schizophrenia have demonstrated a linkage to specific myelin-related gene loci, and that the relative coherence of white matter tracts in schizophrenic patients differs from controls as reflected by a decrease in anisotropy in specific pathways measured by diffusion tensor imaging, and the reduction of myelin content in the temporal and frontal lobes detected by magnetic transfer imaging. These data point to an involvement of oligodendrocyte and myelin in schizophrenia. Importantly, some ultrastructural data from human brain specimens have demonstrated abnormalities of oligodendrocytes and of myelin sheaths in several regions of the brain in patients with schizophrenia, and altered numbers and distribution of oligodendrocytes have been reported in the subcortical white matter of the superior frontal gyrus.

Schizophrenia is a highly multigenic disorder involving the conjugated effects of a constellation of deficits that lead to a common set of functional abnormalities. It is perhaps worth noting that the connectional pathology in schizophrenia may be related to major defects in glutamatergic transmission and may be associated with hypofunction of $N$-methylD-aspartate (NMDA) receptors. It has been proposed that such hypofunction of NMDA receptors would lead to a compensatory release of glutamate that could act on other ionotropic receptor subtypes and induce excitotoxic damage. While such compensatory release of glutamate could have a clear impact on neuronal plasticity and modify synaptic connectivity, it is also important to realize that it may severely affect the integrity of the white matter by acting directly on oligodendrocytes. Mature oligodendrocytes and oligodendrocytes precursor cells are known to possess excitatory glutamate receptors, and stimulation of excitatory axons result in fast receptor-mediated currents in these cells. It is also possible that the glutamate released at the synaptic sites interacts with glial cells through a spillover mechanism. An increased release of glutamate could result in exposing oligodendrocyte to excitotoxic damage. In fact, forebrain oligodendrocytes are highly susceptible to excitotoxicity. This may be an important mechanism in the pathogenesis of schizophrenia that links the alterations in excitatory neurotransmission to the functional integrity of the white matter.

The consistent alterations in neuronal morphology and densities reported in schizophrenia have been related to major developmental abnormalities that lead to a generally disturbed arrangement of specific neuronal and glial populations in key brain regions. Several studies, many using rigorous stereologic methods, have reported variable degrees of changes in neuronal densities or number. The papers included in this issue of Acta Neuropathologica provide an update on several aspects of the neuropathology of schizophrenia. All emphasize the use of stereologic techniques as a standard in quantitative neuropathology. They review modern concepts on the disease pathophysiology and represent our current state of knowledge on the involvement of neocortical, hippocampal and thalamic systems in schizophrenia. In addition, beyond an apparent focus on specific region, these contributions demonstrate the value of system-level, integrated approaches to the study of complex disorders, such as schizophrenia. In the light of the existing literature on genetic alterations and cellular pathology in schizophrenia, these studies provide a summary including quantitative assessment of large-scale abnormalities of gray and white matter organization that take into consideration the pathologic mechanisms affecting specific cortical circuits and white matter tracts. Such data may serve as robust quantitative correlates of the cognitive and psychotic symptoms of schizophrenia. This will be crucial, in combination with molecular biologic and brain imaging studies, to understand further the mechanisms leading to the progression of the disease and permit the development of novel therapeutic or prevention strategies. 